\title{
Compact Surface Plasmon Resonance Sensor for Underwater Chemical Sensing Robot
}

\author{
Yuichi Minagawa, Mari Ohashi, Yoshinori Kagawa, Arata Urimoto, and Hiroshi Ishida \\ Division of Advanced Mechanical Systems Engineering, Tokyo University of Agriculture and Technology, 2-24-16 Nakacho, \\ Koganei, Tokyo 184-8588, Japan \\ Correspondence should be addressed to Hiroshi Ishida; h_ishida@cc.tuat.ac.jp
}

Received 15 November 2016; Accepted 20 February 2017; Published 2 April 2017

Academic Editor: Banshi D. Gupta

Copyright (C) 2017 Yuichi Minagawa et al. This is an open access article distributed under the Creative Commons Attribution License, which permits unrestricted use, distribution, and reproduction in any medium, provided the original work is properly cited.

\begin{abstract}
This paper reports on the development of compact surface plasmon resonance (SPR) sensors for mobile robot olfaction. Underwater robots benefit from olfactory sensing capabilities in various tasks including the search for unexploded ordnance and undersea wreckage. Although the SPR-based chemical sensor is a promising sensing platform, the cumbersome optical setup has been limiting its use on mobile robots. The proposed sensor employs a periodic metal structure formed on a self-assembled layer of polystyrene particles of $200 \mathrm{~nm}$ in diameter. With the grating of this size, SPR can be excited even with a simple LED light source. The change in the absorbance is simply measured using a photodiode. Demonstration of the proposed SPR sensor is provided by mounting the sensors on an underwater crayfish robot that autonomously searches for a chemical source. The fabricated sensor shows linear response to ascorbic acid for a concentration range from 20 to $80 \mathrm{mM}$. Responses of the bare and thiol-coated gold nanostructure to different chemical substances are presented to show the change in the selectivity of the sensor by the coating. Discussions are made on the importance of sample collection for the sensor to attain sensitive chemical detection on a mobile robot.
\end{abstract}

\section{Introduction}

To protect the marine environment and to reduce the risk of hazards, any object containing hazardous and/or toxic chemicals must be localized and removed. The examples of such chemical hazards include heavy oil leaking from undersea wreckage and unexploded ordnance left in the sea [1]. Although searching for those chemical hazards is an extremely laborious task for human divers, underwater robots could be used to accomplish the search-andremoval task if equipped with appropriate sensors. Since the distinctive characteristics of the chemical hazards are the chemical substances they contain, chemical sensors provide more direct evidence of the existence of the chemical hazards than any other types of sensors. If 2,4,6-trinitrotoluene (TNT) is detected, at least some explosives surely exist in the vicinity even though no visual sign is obtained with a camera.

Despite the wide range of potential applications, only a handful of research works were reported on the use of chemical sensors for guiding underwater robots toward the sources of chemical substances. Grasso et al. developed RoboLobster $[2,3]$. Lobsters have keen sense of smell and use it to search for odor sources, for example, food and mates. The primary intention of the authors is to study the olfactory search behavior of lobsters through the process of replicating the animal behavior in the robotic system. Li et al. implemented a moth-inspired chemical plume tracing algorithm in an underwater robot called REMUS and succeeded in locating a source of fluorescent dye from $100 \mathrm{~m}$ [1]. We chose crayfish as a model animal and have been investigating their behavioral mechanism to develop chemical source localization robots. The crayfish robot developed in our group is equipped with active flow generators to enhance the chemical reception and to facilitate the source localization under stagnant flow conditions [4].

However, such robotic applications are extremely challenging for chemical sensors. Ideally, fast sensors with high sensitivity and selectivity are desirable to accomplish the navigation of the robots, although a compromise has to be made in reality at least for some aspects of the sensors. 
Moreover, the chemical sensors must meet the space and power constraints of the mobile robot platforms. In the preceding works on underwater chemical sensing robots, the focuses were mostly placed on the development of effective navigation strategies. Therefore, the sensors and the detection targets were chosen solely for the ease of use in the experiments. RoboLobster was originally equipped with a pair of conductivity sensors whose size and spacing were matched with those of antennules of a lobster [2]. The detection target used in the experiments was salt water released in a fresh water flume. The recent version of RoboLobster is equipped with optical sensors that detect a fluorescent dye (fluorescein) released in water [3]. The target chemical for REMUS robot in the experiments reported in [1] was again a fluorescent dye. A fluorometer was mounted on the robot to detect a trace amount of Rhodamine dye dissolved in water. As described in [4], amperometric sensors were used on our crayfish robot to detect an electrochemically active marker [5]. Ascorbic acid (vitamin C) was chosen as the detection target because of its biocompatibility. It can be mixed in the food for crayfish so that the behavior of the robot can be compared directly with the behavior of the animal in the same experimental setup. To the best of our knowledge, however, none of the robots reported so far is equipped with sensors that can detect a specific chemical substance relevant to real-life applications.

This paper reports on the development of surface plasmon resonance (SPR) sensor to be used for underwater chemical sensing robots. The selectivity of the SPR sensor can be tuned to various chemical substances by placing a coating with an affinity specific to the target chemical [6]. Molecular imprinted polymers are the popular materials for the coating [7]. Antibody-antigen interaction can be also used to provide the sensor with high selectivity $[8,9]$. A highly sensitive TNT sensor is proposed in [8] based on this technique. However, a conventional SPR sensor using a flat gold film is generally cumbersome due to the complicated optical setup and a large built-in temperature regulator. In the most popular optical setup called the Kretschmann configuration, the light must be shed on the gold film through a prism at an exact angle to excite SPR [6]. Metal nanostructures enable the excitation of SPR with much simpler optical setups and, therefore, lead to miniaturization of the whole sensor setup [10-12]. Although the easy excitation of SPR also often results in inferior sensitivity of the sensor to its surface change, nanostructure-based sensor can still find many applications. Here we report a compact SPR sensor consisting of a periodic gold nanostructure, an LED light source, and a photodiode. This paper is an extended version of the work presented at the Seventh International Conference on Machine Learning and Applications [13]. The fabricated SPR sensors were mounted on the crayfish robot, and their chemical sensing capabilities were compared with those of the amperometric electrochemical sensors. The results indicate that not only the sensitivity of the sensors themselves but also how water samples are collected to the sensors greatly affects the chemical sensing capability of the robot. In this paper, a discussion is made on this issue. The fabricated sensor can be also used to test various coatings to tune the selectivity of the sensor for specific applications. The change in the selectivity of the

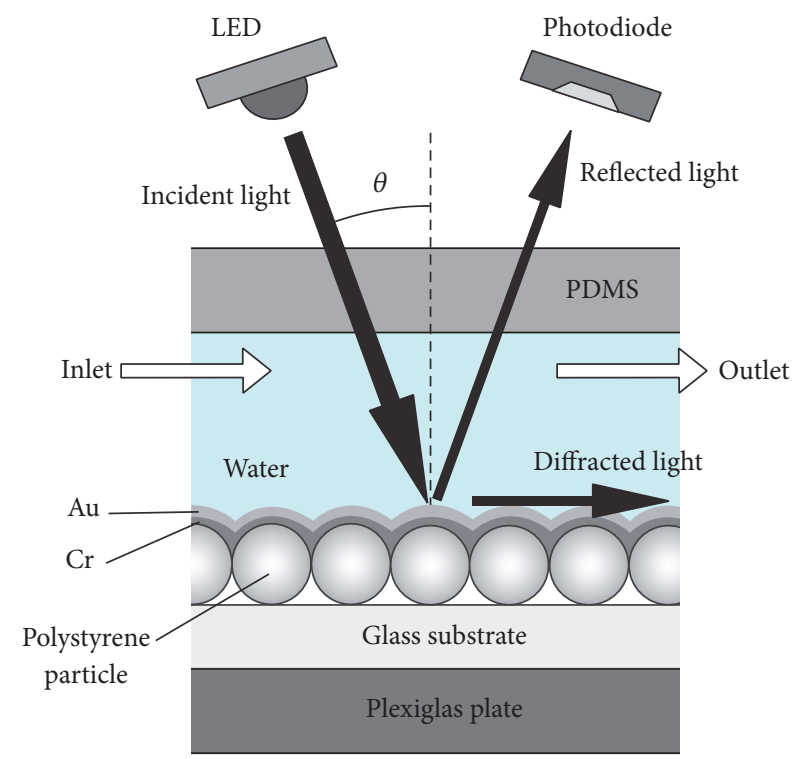

FIGURE 1: Schematic diagram of the SPR sensor.

sensor for a thiol coating is presented in the paper as an example.

\section{Experimental}

2.1. SPR Sensor. The proposed SPR sensor employs a gold nanostructure to achieve the compactness necessary for mobile robot applications. The schematic diagram of the sensor is shown in Figure 1. The gold nanostructure was prepared using the method described in [12]. Firstly, aqueous suspension of polystyrene particles $(200 \mathrm{~nm}$ in diameter) was applied on a glass substrate. As the water evaporated, the polystyrene particles were aligned and a self-assembled monolayer was formed on the glass substrate. The periodic metal nanostructure was then prepared by depositing $10 \mathrm{~nm}$ of chromium and $90 \mathrm{~nm}$ of gold over the polystyrene particles. When the light is shed on the gold nanostructure, a partial component of the diffracted light propagates along the gold nanostructure. SPR is excited when the wave number of the diffracted light matches the wave number of the plasmon. SPR excitation occurs even with an uncollimated, unpolarized light, and, therefore, a simple light source, for example, an LED, can be used. The way of SPR excitation changes when polystyrene particles with a different diameter are used. However, it was reported in [14] that the SPR sensors made with $202 \mathrm{~nm}$ and $356 \mathrm{~nm}$ polystyrene particles showed almost similar response to water vapor.

To confirm the excitation of SPR, the absorbance spectrum of the gold nanostructure was measured using a tungsten-halogen lamp (LS-1, Ocean Optics) and a spectrometer (USB4000, Ocean Optics). The fabricated gold nanostructure was placed in a flow-through cell with a volume of $94.2 \mu \mathrm{L}$. The white light from the light source was shed through a transparent Plexiglas ceiling of the flow-through cell. The incident angle of the light, $\theta$, was set to $20^{\circ}$. The measured absorbance spectra are shown in Figure 2. The peak in the absorbance spectrum corresponds to the wavelength 


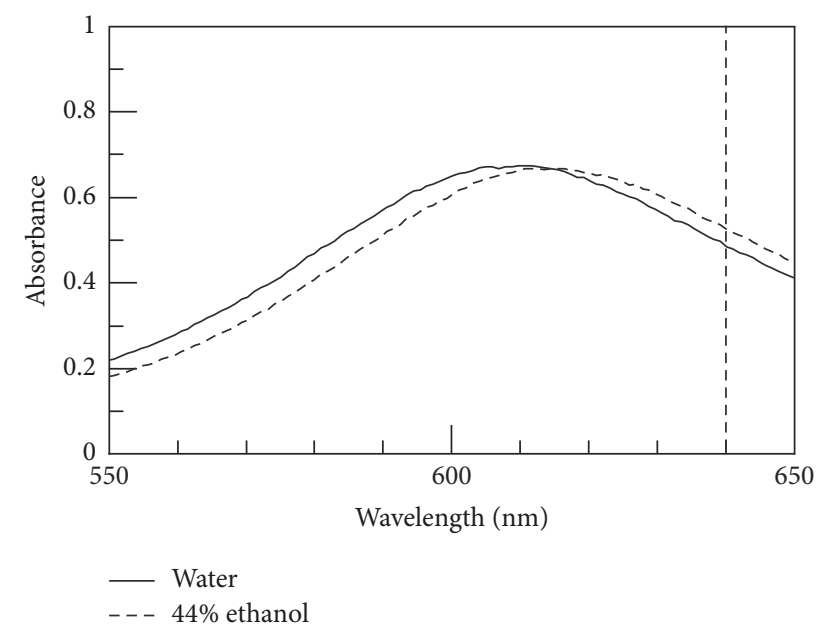

FIgURE 2: Absorbance spectra of the gold nanostructure. The vertical dashed line indicates the wavelength of the LED at which the change in the reflectance was measured.

at which SPR is excited. The change in the refractive index of the material in contact with the gold surface causes the shift in the wavelength of the SPR excitation. When the flow-through cell was filled with water, SPR was excited at $610 \mathrm{~nm}$. When the water was replaced with aqueous solution of ethanol, the absorbance peak was shifted to a longer wavelength because of the larger refractive index of ethanol.

When the gold nanostructure is exposed to varying concentration of the analyte, the refractive index of the solution in the flow-through cell changes accordingly. This results in the small change in the actual incident angle of the light to the gold nanostructure and the slight shift in the location of the light spot. Moreover, a coating is placed on the gold nanostructure to improve the selectivity of the sensor. Therefore, it is desirable to measure the absorbance spectrum from the backside of the gold nanostructure. However, the absorbance spectrum measured from the backside shows a complicated behavior. The absorbance peak caused by the SPR excitation is overlapped with the peaks and dips caused by Bragg diffraction at the polystyrene particles. The variation in the analyte concentration causes the change in the refractive index of the solution that penetrates into the small gaps between the polystyrene particles and the glass substrate. When the absorbance spectrum was measured from the backside, the change in the SPR excitation wavelength was masked with the shift in the dips and peaks of the absorbance spectrum caused by Bragg diffraction. For this reason, the absorbance was measured in the experiments from the front side of the gold nanostructure. The influence of the change in the optical path due to the variation of the refractive index is so far indiscernible in the experimental results.

Figure 3 shows the fabricated SPR sensor. In the SPRbased humidity sensor reported in [12], the shift in the absorbance spectrum of a metal nanostructure was measured using a halogen lamp and a spectrometer. To realize an even smaller sensor, the sensor shown in Figure 3 uses an LED with a wavelength of $640 \mathrm{~nm}$ as a light source. The concentration of a chemical substance is measured by detecting the change

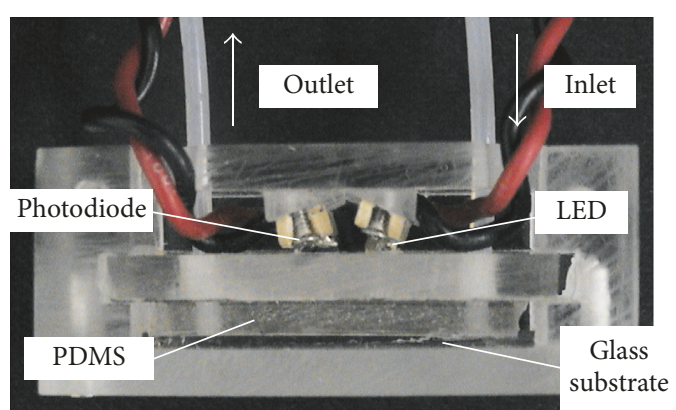

FIGURE 3: Compact SPR sensor for underwater chemical sensing robot.

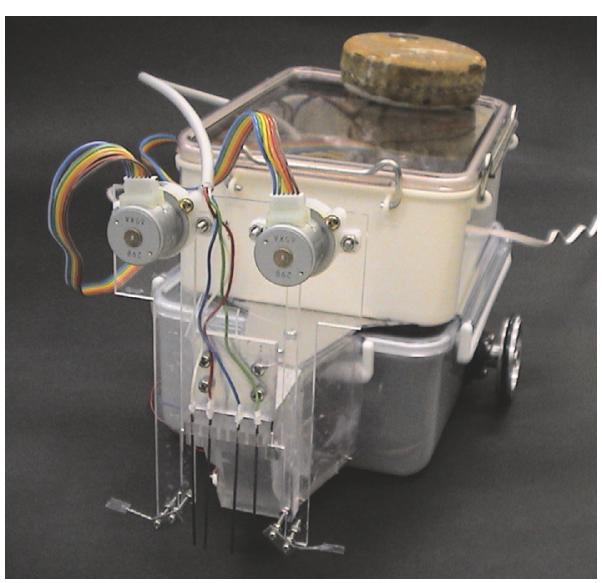

FIGURE 4: Crayfish robot equipped with electrochemical sensors and active fanning devices.

in the intensity of the reflected light using a photodiode. As shown in Figure 2, the increase in the concentration of a chemical substance causes the increase in the absorbance at $640 \mathrm{~nm}$. Therefore, the intensity of the reflected light decreases with the concentration of the chemical. In the experiments, the SPR sensors were mounted on a crayfish robot, and ascorbic acid was used as the detection target as in the previous paper [4]. The gold nanostructure was coated with a hydrophilic self-assembled monolayer of 11-mercapto1-undecanol to enhance the affinity to organic molecules. The flow-through cell was constructed by placing a flow channel made of PDMS (polydimethylsiloxane) on the gold nanostructure. The size of the sensor setup shown in Figure 3 is $40 \mathrm{~mm} \times 23 \mathrm{~mm} \times 20 \mathrm{~mm}$. The water sample is led into the flow channel at a rate of $0.8 \mathrm{~mL} / \mathrm{min}$ by a small peristaltic pump $(12 \mathrm{~mm} \times 30 \mathrm{~mm} \times 14 \mathrm{~mm})$. The volume of the flow channel is $14 \mu \mathrm{L}$. The water sample can be quickly replaced even with a small flow rate.

2.2. Crayfish Robot. Two SPR sensors were fabricated and mounted on the crayfish robot shown in Figure 4 to test their responses. This $350 \mathrm{~mm}$ long robot had accomplished chemical source localization by using the amperometric electrochemical sensors [4]. In the original setup, four carbon rods $(\phi 0.9 \mathrm{~mm} \times 60 \mathrm{~mm})$ were placed at the front part of the robot head and were used as the working electrodes. They 


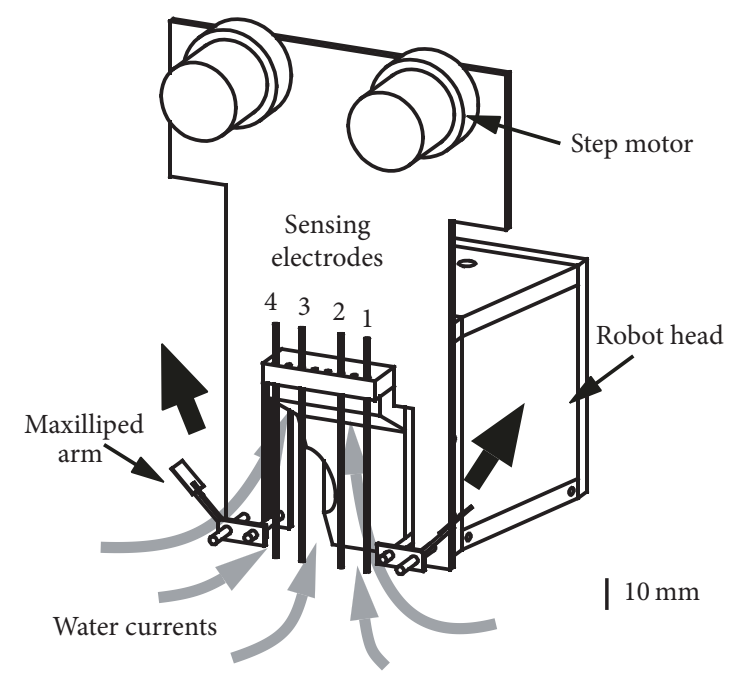

FIgURE 5: Head part of the crayfish robot. The upward water currents (black arrows) generated by waving the maxilliped arms induce inflow (gray arrows) that draws water samples from the surroundings to the sensors.

were numbered from the left to the right of the robot, as shown in Figure 5. A silver reference electrode and a stainlesssteel counter electrode were placed on the bottom of the robot head and shared by the four working electrodes. The potential of the working electrodes against the reference electrode was set to $0.7 \mathrm{~V}$, and the current caused by the oxidation of ascorbic acid was measured.

The crayfish robot has a pair of arms mimicking the maxillipeds of a crayfish and actively generates water currents by waving them. Crayfish (Procambarus clarkii) which prefer to live in still water search for food by using their olfaction. Odor molecules released from food stay in the vicinity of the food under stagnant flow conditions [15]. Since the smell of food hardly reaches their chemoreceptor organs (antennules), crayfish generate water currents by waving their fan organs (exopodites of maxillipeds) to draw odor molecules $[15,16]$. A crayfish has three pairs of maxillipeds around the mouth opening under the antennules. Upward water currents generated by waving of the maxillipeds induce inflow of water samples from the surroundings to their antennules. Similarly, the crayfish robot actively generates upward water currents by waving the maxilliped arms vertically by $90^{\circ}$ at $5 \mathrm{~Hz}$ using the step motors. The inflow induced by these water currents draws water samples from the surroundings to the sensing electrodes. If a source of a chemical substance is placed at the left of the robot, the chemical drawn from the source mostly reaches sensors 1 and 2 in Figure 5. When the source is at the right of the robot, the chemical is drawn to sensors 3 and 4. Consequently, the direction of the chemical source can be determined by comparing the responses of the four sensing electrodes. The crayfish robot had accomplished chemical source localization by simply comparing the sensor responses and moving in the direction of the sensor with the largest response.

In the experiments to test the SPR sensors, the four electrochemical sensors were replaced with two SPR sensors.

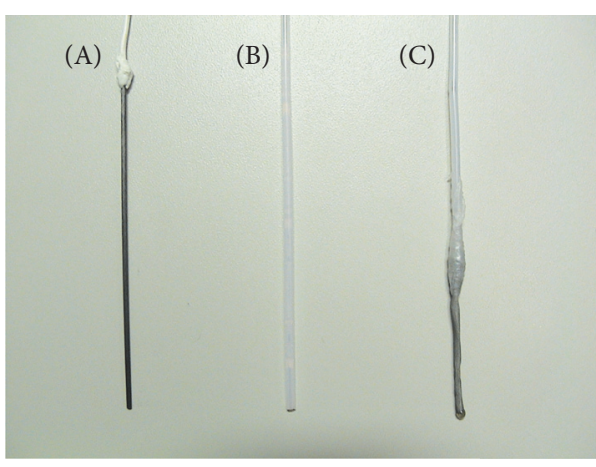

FIgURE 6: (A) Carbon electrode of the amperometric sensor. (B) Suction tube of the SPR sensor. (C) Suction tube with a metal mesh tip.

A water sample was sucked into each SPR sensor using a pump through a PTFE (polytetrafluoroethylene) tube with an inner diameter of $0.8 \mathrm{~mm}$ and an outer diameter of $1.6 \mathrm{~mm}$ (Figure 6). The inlet of the suction tube for the left sensor (sensor L) was placed at the same position as the lower tip of sensing electrode 1. Similarly, the inlet for the right sensor (sensor R) was positioned at the location of sensing electrode 4 . The distance between these tubes was $25 \mathrm{~mm}$, and their tips were pointed downward. Similarly to other adsorption-based chemical sensors, the changes in the temperature cause variations in the SPR sensor response. The amount of chemical substances that adsorbs on to the SPR sensor surface decreases with temperature. Conventional SPR equipment generally has a built-in temperature regulator to achieve precise measurement. However, the temperature regulation may not be always required for the robot. As long as both sensors are exposed to the same ambient temperature, the direction toward a chemical source can be determined by the left-and-right comparison of the sensor responses. We fabricated several sensors and they all show similar responses. In the experiments, the change in the photocurrent of each SPR sensor from its baseline was measured in order to alleviate the problems of the temperature dependence of the sensors, the individual differences between the sensors, and their baseline drift. The baseline value of the photocurrent of each sensor was recorded immediately before the start of each experiment.

\section{Results}

3.1. Response of SPR Sensor to Ascorbic Acid. The calibration curve of the SPR sensor was measured before testing the sensor on the crayfish robot. Figure 7 shows the sensor response to ascorbic acid. Aqueous solutions of ascorbic acid with different concentrations were prepared in vials. A threeway solenoid valve was used to switch the solutions, and the short-circuit photocurrent of the photodiode was measured. The inlet tubes of the solenoid valves were first put in the vials containing distilled water and $20 \mathrm{mM}$ ascorbic acid solution. After switching the valve from water to $20 \mathrm{mM}$ solution, the tube used to suck the distilled water was moved manually to the vial containing $40 \mathrm{mM}$ ascorbic acid solution. When the 


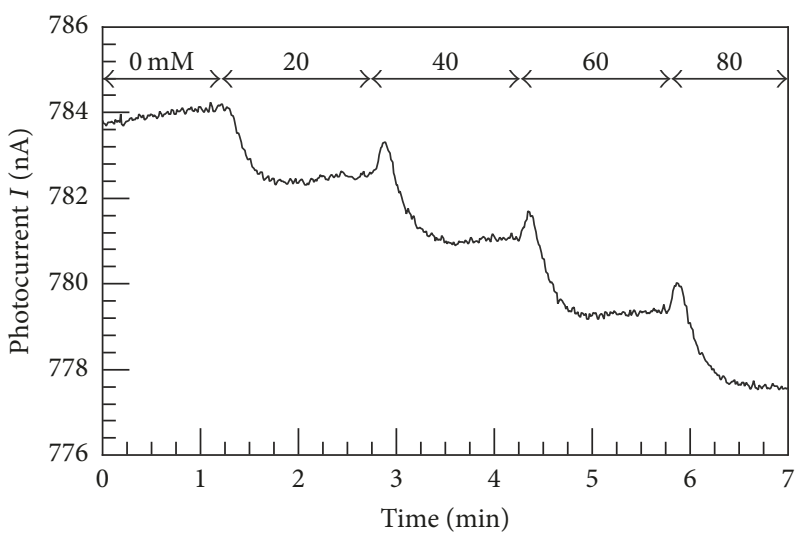

FIGURE 7: Time course of the SPR sensor response to ascorbic acid solutions with different concentrations $(0-80 \mathrm{mM})$.

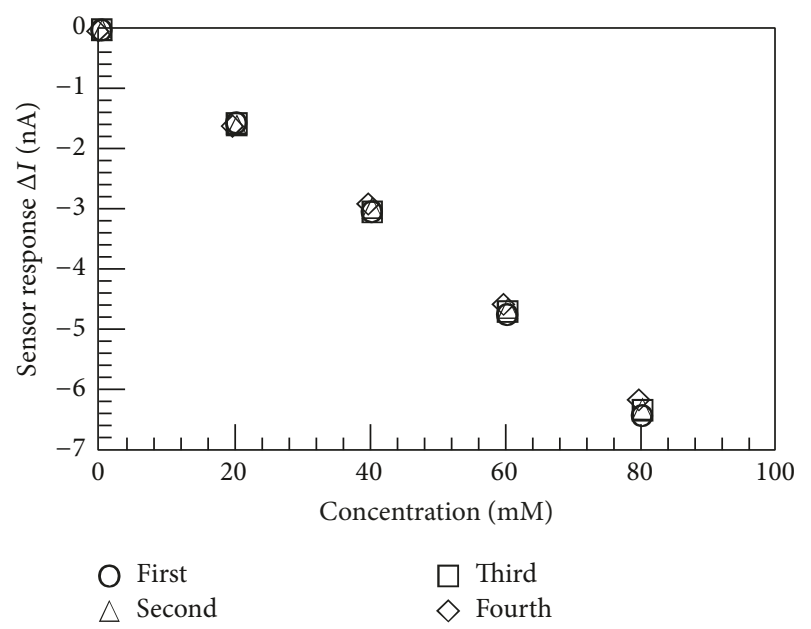

FIGURE 8: Dependence of the SPR sensor response on ascorbic acid concentration obtained from four repeated measurements.

valve was switched from $20 \mathrm{mM}$ solution to $40 \mathrm{mM}$, a small amount of the distilled water that remained in the tube was first sucked into the flow channel of the sensor. The small increase in the photocurrent observed when switching the solutions was caused in this way. The rest of the measurement was conducted similarly.

The same measurement was repeated four times, and the results are shown in Figure 8. The sensor response, $\Delta I$, was defined as the change in the short-circuit photocurrent of the photodiode from the initial state in which the sensor was exposed to distilled water. The sensor showed good reproducibility, and its response was found to be linear in this range of concentration.

3.2. Detection of Chemical Substance Released in Water. To compare the responses of the amperometric electrochemical sensors and the SPR sensors, the crayfish robot was placed in a water container $(500 \mathrm{~mm} \times 500 \mathrm{~mm} \times 155 \mathrm{~mm})$ made of transparent Plexiglas. Since a certain amount of supporting electrolyte is required for proper operation of the electrochemical sensors, the container was filled with salt water up

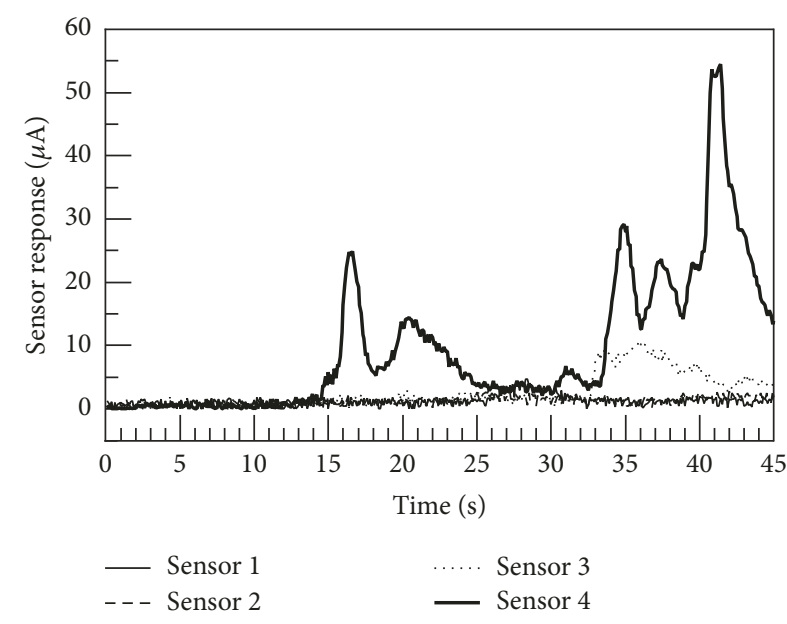

FIGURE 9: Responses of the amperometric electrochemical sensors observed when the chemical source was placed at the right front of the robot.

to a depth of $100 \mathrm{~mm}$. The concentration of salt (sodium chloride) was adjusted to be in the rage of $0.1 \mathrm{M}$ to $0.485 \mathrm{M}$ [4]. When the SPR sensors were used in the experiments, the container was filled with fresh water to the same depth. Ascorbic acid solution was released from a stainless-steel tube with an inner diameter of $4.0 \mathrm{~mm}$ and an outer diameter of $4.6 \mathrm{~mm}$. A small amount of a fluorescent dye $(10 \mathrm{mg} / \mathrm{L}$ of Rhodamine 6G) was added to the ascorbic acid solution to enable the visual observation of the ascorbic acid distribution.

The responses of the amperometric electrochemical sensors are shown in Figure 9. The tube for the chemical release was placed $20 \mathrm{~mm}$ to the front from sensor 4 . The tip of the tube was pointed upward, and ascorbic acid solution with a concentration of $10 \mathrm{mM}$ was released at a height of $30 \mathrm{~mm}$. The release rate was set to $1 \mathrm{~mL} / \mathrm{min}$. The chemical release was started when the waving of the maxilliped arms and measurement of the sensor responses were initiated. The released chemical was drawn from the source to the sensors by actively generating water currents. In Figure 9, the response of sensor 4 started to increase at $14 \mathrm{~s}$ after the start of the waving of the arms. The sensor response of $50 \mu \mathrm{A}$ corresponds to the ascorbic acid concentration of approximately $0.05 \mathrm{mM}$. The patch of the released chemical is mostly drawn to the sensor closer to the source. Therefore, the largest response was obtained from sensor 4 .

In the experiments for testing the SPR sensors mounted on the crayfish robot, the concentration of ascorbic acid solution was increased to $1.5 \mathrm{M}$ due to the lower sensitivity of the SPR sensors. Because of the increased concentration, the mismatch in the density between the ascorbic acid solution and the background water became larger. Therefore, the tip of the tube for the chemical release was tilted by $50^{\circ}$ to the robot and was raised to a height of $70 \mathrm{~mm}$. The inner diameter of the tube was $4 \mathrm{~mm}$ and the outer diameter was $6 \mathrm{~mm}$. The rate of chemical release was set to $1.9 \mathrm{~mL} / \mathrm{min}$. At this flow rate, the distribution of the released chemical was found not affected by the orientation of the tube for the chemical release. The released chemical was spilling out from the tip of the tube and was flowing down vertically. The chemical release 


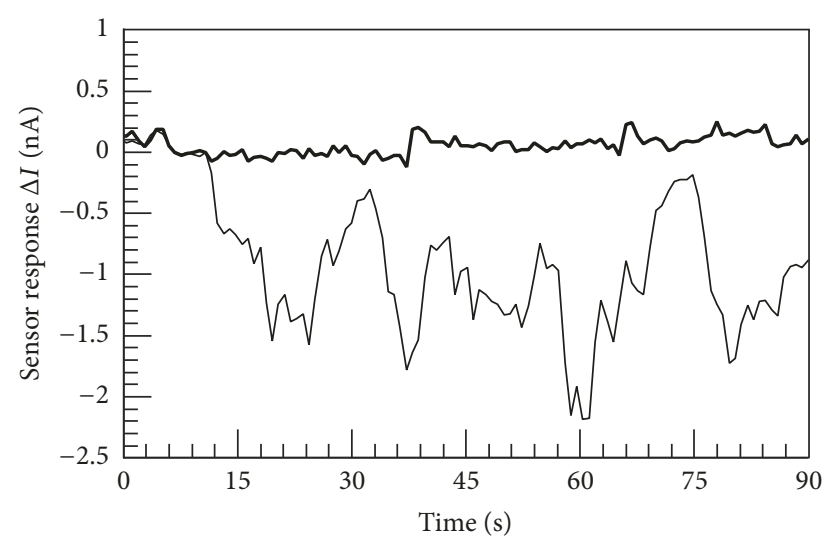

- Sensor L

FIgURE 10: Responses of the SPR sensors observed when the chemical source was placed in front of sensor L.

was again started after the measurement of the SPR sensor responses and waving of the maxilliped arms were started. In the experiments presented in this paper, the SPR sensors were powered not by the batteries on-board the robot but by an external DC power supply. Future work should be addressed to see if stability of the same level is attained for the LED light sources of the sensors when powered by the batteries. The flow-through cells and the electronic circuits of the sensors were placed in metal mesh shields to protect them from the noise induced by the motors of the maxilliped arms.

Figure 10 shows a typical sensor response curves obtained when the tube for the chemical release was placed $20 \mathrm{~mm}$ to the front from sensor L. The response to the released chemical was observed only from sensor L. When the chemical was released from a point $20 \mathrm{~mm}$ to the front from sensor $\mathrm{R}$, the response to the chemical was observed only from sensor $\mathrm{R}$, as shown in Figure 11. The experiment was repeated five times for each source location, and the sensor response was always observed only from the sensor closer to the chemical source. In this simplified situation, the direction of the chemical source can be easily determined by comparing the left and right sensor responses. The photocurrent of $2 \mathrm{nA}$ in Figures 10 and 11 corresponds to the ascorbic acid concentration of approximately $20 \mathrm{mM}$. Ascorbic acid solution with a concentration of $1.5 \mathrm{M}$ was released from the source. To detect this chemical, however, the robot had to draw the released chemical to its sensors with the help of the water currents generated with the maxilliped arms. Moreover, the chemical had to be drawn into the suction tube to be delivered to the SPR sensor. The released chemical was significantly diluted in those processes.

3.3. Sampling of the Released Chemical. The results presented in the previous section imply that collecting water samples efficiently to the sensors is also a key to enable the detection of trace chemicals dissolved in water. The electrochemical sensors mounted on the crayfish robot have carbon rod working electrodes. Each electrode responds if the chemical reaches any point on the large surface area of the electrode.

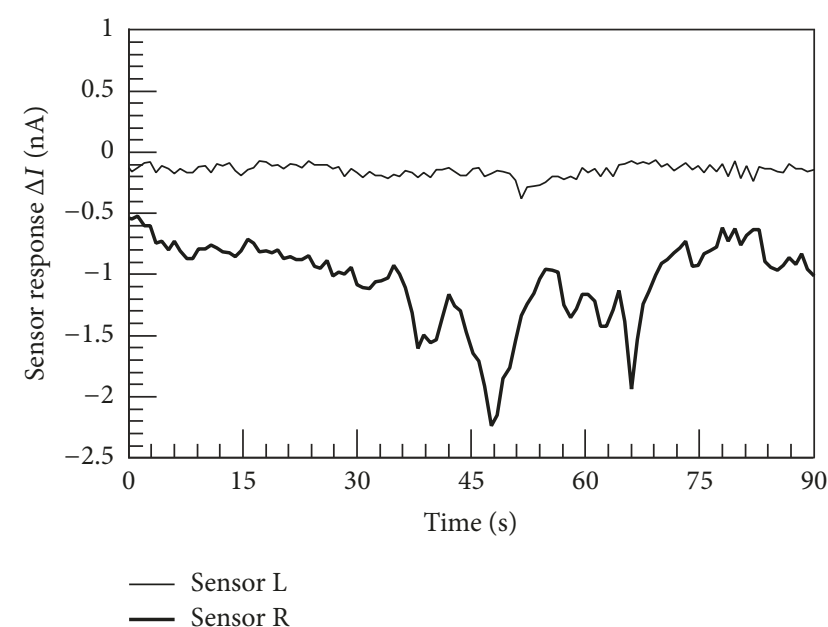

FIGURE 11: Responses of the SPR sensors observed when the chemical source was placed in front of sensor R.

The robot was able to draw and detect the chemical substance from almost all directions. When the SPR sensors were mounted on the crayfish robot, the robot succeeded in detecting the chemical for two source locations as shown in Figures 10 and 11. However, no sensor response was observed if the source was moved by a few millimeters to one side or the other, although the chemical was successfully drawn by the actively generated water currents to the vicinity of the suction tubes of the sensors. It was found from visual observation of the released chemical that the SPR sensor was collecting the water sample only from a small volume around the tip of its suction tube. Therefore, experiments were conducted to quantify the size of the suction volume.

Firstly, the suction tube of the SPR sensor and the tube for the chemical release were placed as shown in Figure 12(a). The tube for the chemical release has an inner diameter of $4 \mathrm{~mm}$ and an outer diameter of $6 \mathrm{~mm}$. $1.5 \mathrm{M}$ ascorbic acid solution containing a small amount of Rhodamine $6 \mathrm{G}$ dye $(10 \mathrm{mg} / \mathrm{L})$ was released at a rate of $1.9 \mathrm{~mL} / \mathrm{min}$. The response of the SPR sensor was measured while changing the position of the suction tube. Visual observation confirmed that a plume of ascorbic acid with a width of $1 \mathrm{~mm}$ was extending down from the tip of the tube due to the higher density of the solution. If the tip of the suction tube was out of the plume, that is, if $d_{\mathrm{a}}>0.5 \mathrm{~mm}$, no sensor response was observed. Secondly, the suction tube of the SPR sensor and the tube for the chemical release were placed as shown in Figure 12(b). $150 \mathrm{mM}$ ascorbic acid solution was released at the rate of $1.9 \mathrm{~mL} / \mathrm{min}$. The released solution came to a height of $1 \mathrm{~mm}$ up from the tube for the chemical release. The sensor response was obtained only when the tip of the suction tube was brought close enough to the chemical source $\left(d_{\mathrm{b}}<2 \mathrm{~mm}\right)$. From the fluid dynamics perspective, the tip of the suction tube can be regarded as a point sink. In this case, the inflow generated by the suction becomes almost spherically symmetric. The flow velocity decays as the inverse square of the distance from the point sink. Therefore, the volume over which the sensor can collect water sample is extremely small. The size of the volume or the area over which the sensor can collect 


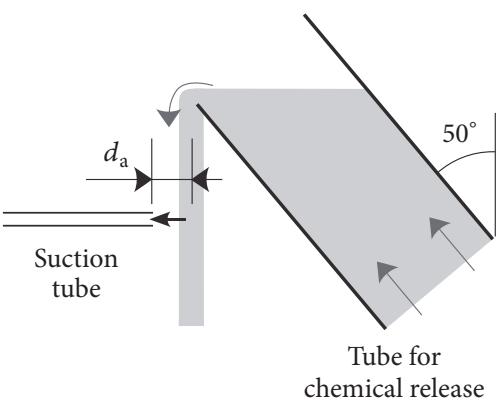

(a)

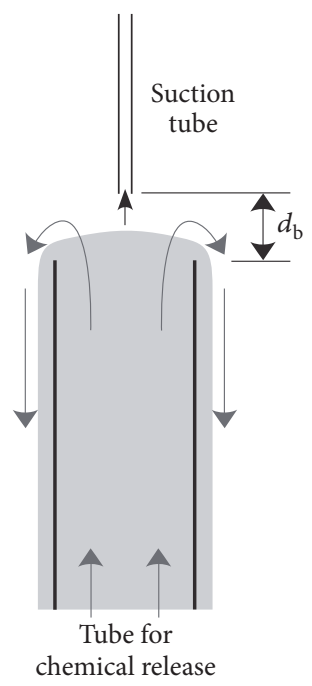

(b)

FIgURE 12: Schematic diagrams of the experimental setups for examining the sampling volume of the SPR sensor. (a) $1.5 \mathrm{M}$ ascorbic acid solution was released from a tube tilted by $50^{\circ}$. The suction tube of the sensor was placed horizontally. (b) $150 \mathrm{mM}$ ascorbic acid solution was released from a tube with its tip pointing upward. The tip of the suction tube of the sensor was pointed downward. Gray arrows indicate the flow of the ascorbic acid solution.

chemical information turns out to be an important feature for the underwater chemical sensing robot.

One way to increase the volume of chemical reception is to use a meshed tube. The tube shown in Figure 6(C) was fabricated by attaching a roll of a stainless-steel 400-mesh screen to the tip of a PTFE tube. Visual observation confirmed that the chemical patch reaching at any point of the stainless-steel mesh was sucked into the tube and delivered to the flowthrough cell. We expected that the SPR sensor equipped with this mesh suction tube would have better chemical reception capability. However, when the same experiments as those described in Section 3.2 were conducted, no sensor response was obtained due to the dilution of the sucked chemical. The width of the chemical plume was only a few millimeters whereas the length of the mesh tube was $20 \mathrm{~mm}$. Even when the plume was touching the mesh tube, the chemical was sucked together with surrounding fresh water and was diluted before delivered to the SPR sensor. Increasing the flow rate for water suction would also bring a similar result. A small sensor mounted in a flow-through cell has an inherent disadvantage over a sensor with a large surface area.

3.4. Change in the Selectivity of the Sensor by Coating. One of the virtues of the SPR sensors is the capability of tuning its selectivity by depositing a selective coating on it. As described in Section 2.1, the gold nanostructure in our sensor was coated by 11-mercapto-1-undecanol. The response of the SPR sensor before and after the deposition of this thiol coating is compared in Figure 13. Eight chemical substances listed in Table 1 were used in the experiments. $10 \mathrm{mM}$ aqueous solution of each chemical substance was prepared, and the response of the SPR sensor was measured by sucking the solution into the flow-through cell. For isobutyric acid, propionic acid, and ascorbic acid, the concentration of the solution was increased
TABLE 1: Eight chemical substances used to test the selectivity of the thiol coating.

\begin{tabular}{lc}
\hline Symbol & Name \\
\hline A & Trans-2-hexenal \\
B & Isobutyric acid \\
C & Ethyl valerate \\
D & Propionic acid \\
E & $1-$ Hexanol \\
F & $1-$ Butanol \\
G & Butyl propionate \\
H & Ascorbic acid \\
\hline
\end{tabular}

to $20 \mathrm{mM}$. The experiments were repeated three times for each chemical substance. As shown in Figure 13, the response of the SPR sensor to acids (isobutyric acid, propinonic acid, and ascorbic acid) was increased by putting the thiol layer on the sensor surface whereas the response to other chemical substances was decreased. These changes are attributed to the hydrophilic hydroxyl group on the surface of the thiol layer. There are ways to further improve the selectivity of the sensor, for example, coating the sensor with a molecular imprinted polymer and immobilizing antibodies on the sensor surface. The fabricated sensor can be used as a test bed to try various coatings.

\section{Conclusions}

Compact SPR sensors were developed. The proposed sensor uses a gold nanostructure for SPR excitation and reacts reproducibly to the changes in the concentration of a target chemical. The size of the fabricated sensor was $40 \mathrm{~mm} \times$ $23 \mathrm{~mm} \times 20 \mathrm{~mm}$ and can be easily mounted on a small mobile 


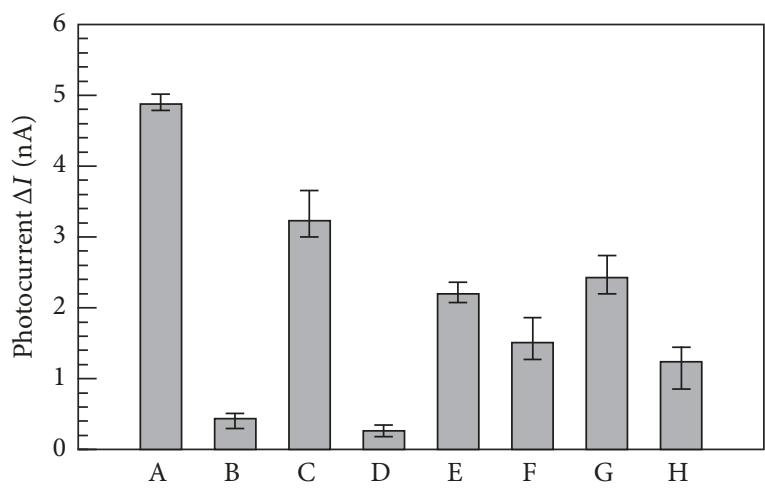

(a)

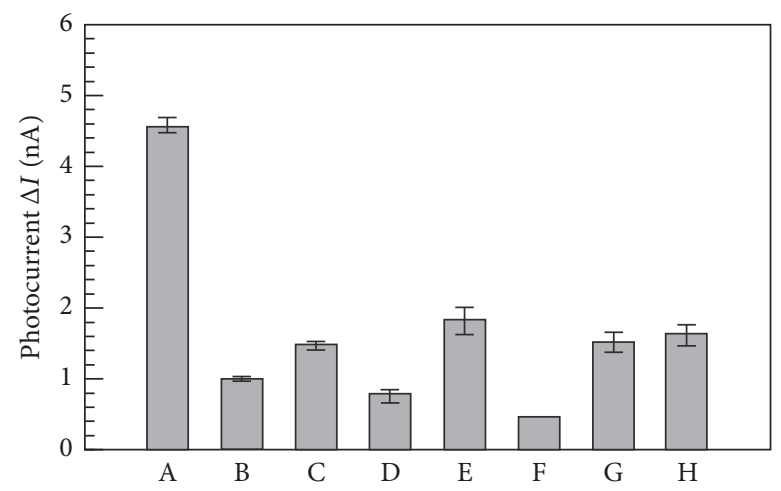

(b)

FIGURE 13: Response of the SPR sensor to eight chemical substances listed in Table 1. Each bar shows the mean photocurrent for three repeated experiments. Error bars show the minimum and maximum of the three measurements. (a) Bare gold nanostructure. (b) Gold nanostructure coated with a self-assembled monolayer of 11-mercapto-1-undecanol.

robot. The indication of the direction of a chemical source was successfully obtained from the responses of the SPR sensors mounted on the crayfish robot. Future work will be addressed to increase the sensitivity of the sensor by using a stronger light source as a laser diode. Various sensor coatings will also be tested to attain better sensitivity and selectivity.

The measurements obtained with the sensors on the crayfish robot suggest that not only the sensitivity of the sensor but also the method for sample collection affects the sensing capability of the robot. Although the water currents generated by the maxilliped arms brought the water samples containing the target chemical to the vicinity of the suction tube, only those coming within a small radius from the tube opening were sucked to the SPR sensor. On the contrary, the electrochemical sensor showed at least some response whenever the chemical was drawn to the vicinity of any part of the electrode because of its large surface area. This implies that better chemical sensing capability is attained by having a chemical sensor with a large surface area rather than trying to collect large volume of water samples to the sensor housed in a flow-through cell. An array of small sensors distributed over the robot body would also have a large effective surface area. It would be interesting to further investigate its biological relevance since some aquatic animals are known to have chemoreceptors distributed over their bodies [17].

Despite the difficulties in attaining sensitive chemical detection, the flow-through cell has several advantages, for example, better sensor protection. If an array of several sensors is placed in a single flow-through cell, those sensors are exposed to the same water sample. Recognition of the response patterns and therefore the discrimination of the detected chemical would become easier compared to the sensor array distributed over the robot body. Using multimodal sensors might be a practical solution. An underwater robot can use electrochemical sensors to track a chemical trail. When the robot comes close enough to the chemical source, the SPR sensors housed in a flow-through cell can be used to analyze the detected chemical substance in more detail.

\section{Conflicts of Interest}

The authors declare that they have no conflicts of interest regarding the publication of this paper.

\section{Acknowledgments}

The authors would like to thank Professor Norihiro Umeda and Dr. Takayuki Numata for their help in the fabrication of the gold nanostructure. This work was supported in part by JSPS KAKENHI Grant Numbers 18360120, 21360113, and 25289055.

\section{References}

[1] W. Li, J. A. Farrell, S. Pang, and R. M. Arrieta, "Moth-inspired chemical plume tracing on an autonomous underwater vehicle," IEEE Transactions on Robotics, vol. 22, no. 2, pp. 292-307, 2006.

[2] F. W. Grasso, T. R. Consi, D. C. Mountain, and J. Atema, "Biomimetic robot lobster performs chemo-orientation in turbulence using a pair of spatially separated sensors: progress and challenges," Robotics and Autonomous Systems, vol. 30, no. 1-2, pp. 115-131, 2000.

[3] F. W. Grasso and J. Atema, "Integration of flow and chemical sensing for guidance of autonomous marine robots in turbulent flows," Environmental Fluid Mechanics, vol. 2, no. 1-2, pp. 95-114, 2002.

[4] M. Ohashi, Y. Kagawa, T. Nakatsuka, and H. Ishida, "Crayfish robot that generates flow field to enhance chemical reception," Journal of Sensor Technology, vol. 2, no. 4, pp. 185-195, 2012.

[5] T. Kikas, H. Ishida, and J. Janata, "Chemical plume tracking. 3. Ascorbic Acid: a biologically relevant marker," Analytical Chemistry, vol. 74, no. 15, pp. 3605-3610, 2002.

[6] T. C. Pearce, S. S. Schiffman, H. T. Nagle, and J. W. Gardner, Eds., Handbook of Machine Olfaction: Electronic Nose Technology, Wiley-VCH, Weinheim, Germany, 2003.

[7] A. L. Hillberg and M. Tabrizian, "Biomolecule imprinting: developments in mimicking dynamic natural recognition systems," IRBM, vol. 29, no. 2-3, pp. 89-104, 2008. 
[8] D. R. Shankaran, K. V. Gobi, T. Sakai et al., "A novel surface plasmon resonance immunosensor for 2,4,6-trinitrotoluene (TNT) based on indirect competitive immunoreaction: a promising approach for on-site landmine detection," IEEE Sensors Journal, vol. 5, no. 4, pp. 616-621, 2005.

[9] V. Nanduri, A. K. Bhunia, S.-I. Tu, G. C. Paoli, and J. D. Brewster, "SPR biosensor for the detection of L. monocytogenes using phage-displayed antibody," Biosensors and Bioelectronics, vol. 23, no. 2, pp. 248-252, 2007.

[10] T. Okamoto, I. Yamaguchi, and T. Kobayashi, "Local plasmon sensor with gold colloid monolayers deposited upon glass substrates," Optics Letters, vol. 25, no. 6, pp. 372-374, 2000.

[11] K. Mitsui, Y. Handa, and K. Kajikawa, "Optical fiber affinity biosensor based on localized surface plasmon resonance," Applied Physics Letters, vol. 85, no. 18, pp. 4231-4233, 2004.

[12] T. Numata, Y. Otani, and N. Umeda, "Optical dew sensor using surface plasmon resonance of periodic Ag nanostructure," Japanese Journal of Applied Physics, vol. 45, no. 29-32, pp. L810L813, 2006.

[13] M. Ohashi, Y. Minagawa, Y. Myoren, and H. Ishida, "Crayfish robot employing flow induced by waving to locate a chemical source," in Proceedings of the 7th International Conference on Machine Learning and Applications (ICMLA '08), pp. 482-488, IEEE, San Diego, Calif, USA, December 2008.

[14] T. Numata, Y. Otani, and N. Umeda, "Meso-porous membrane of noble metal for surface plasmon resonance gas sensors," Journal of Materials Science, vol. 42, no. 3, pp. 1050-1053, 2007.

[15] T. Breithaupt, "Fan organs of crayfish enhance chemical information flow," Biological Bulletin, vol. 200, no. 2, pp. 150-154, 2001.

[16] P. Denissenko, S. Lukaschuk, and T. Breithaupt, "The flow generated by an active olfactory system of the red swamp crayfish," Journal of Experimental Biology, vol. 210, no. 23, pp. 4083-4091, 2007.

[17] T. A. Keller, I. Powell, and M. J. Weissburg, "Role of olfactory appendages in chemically mediated orientation of blue crabs," Marine Ecology Progress Series, vol. 261, pp. 217-231, 2003. 


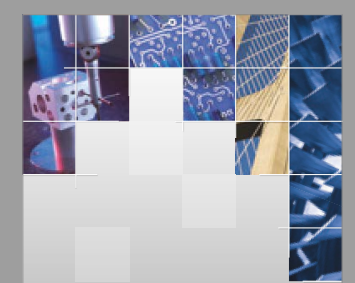

\section{Enfincering}
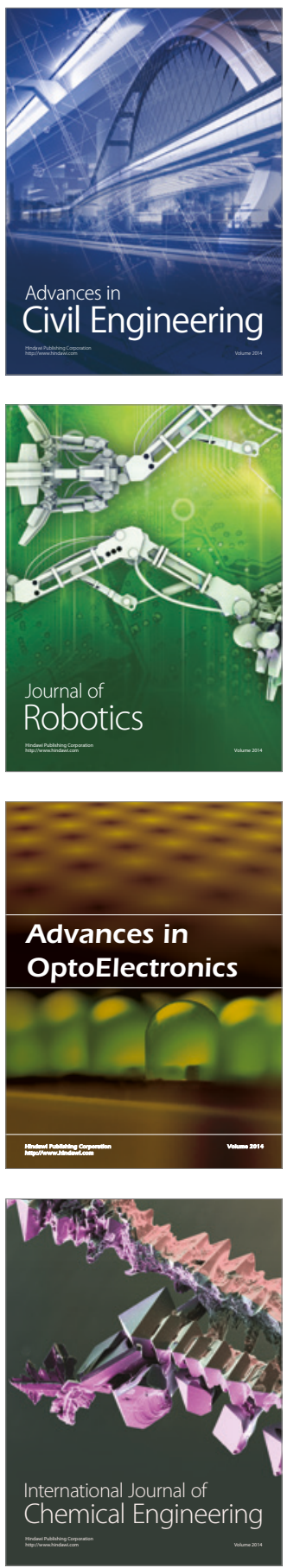

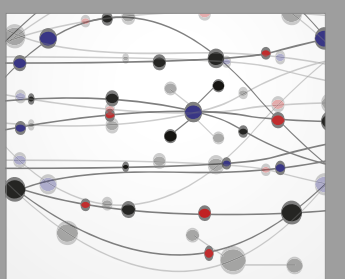

The Scientific World Journal

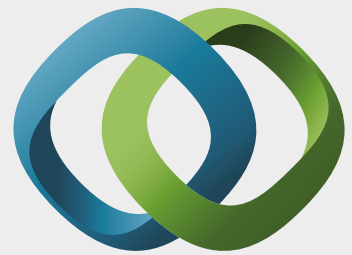

\section{Hindawi}

Submit your manuscripts at

https://www.hindawi.com
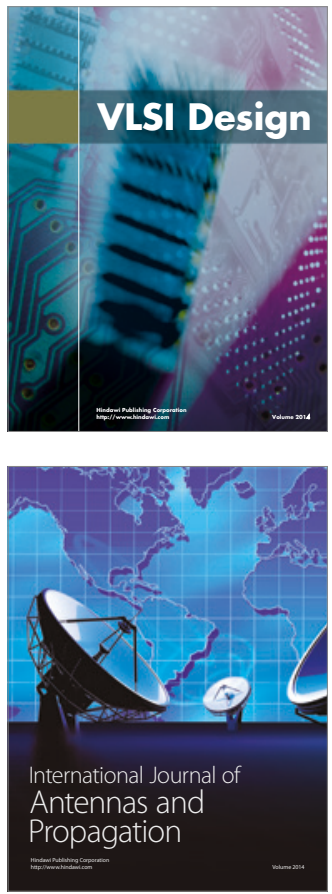

\section{Rotating}

Machinery
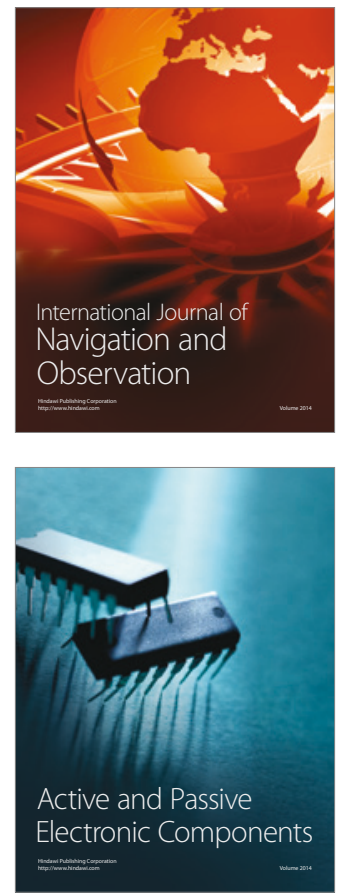
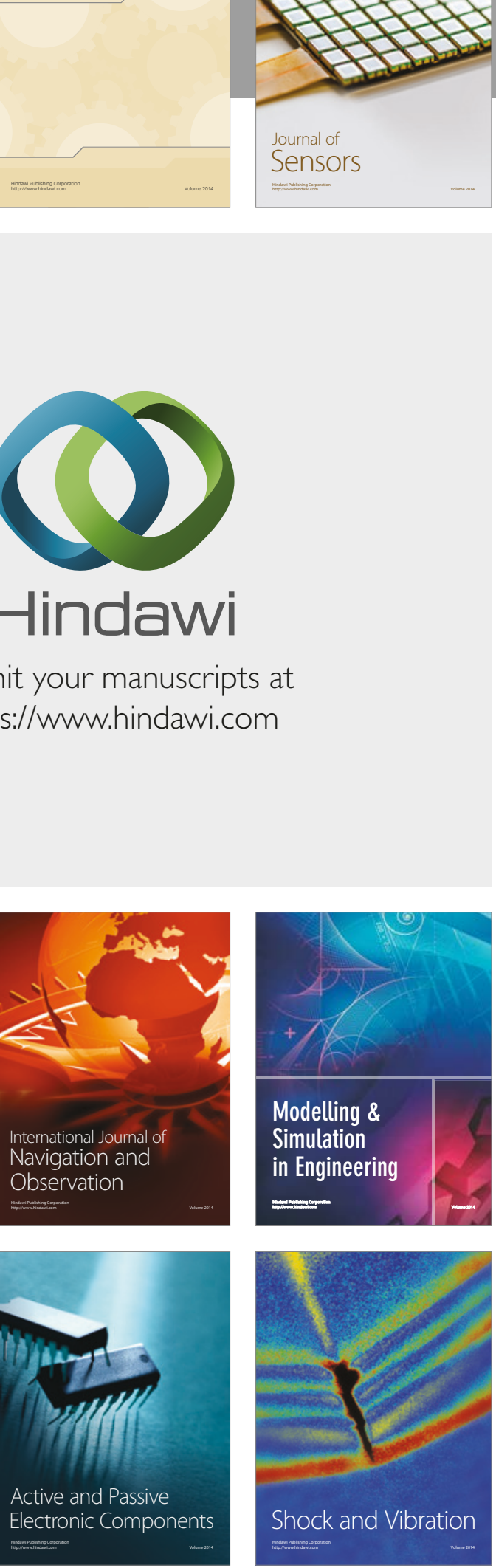
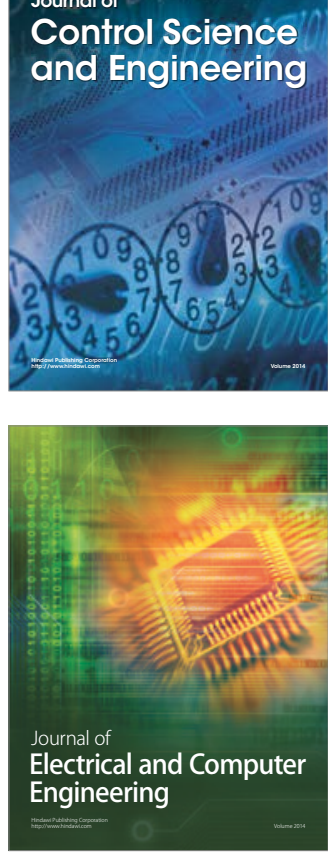

Distributed

Journal of

Control Science

and Engineering
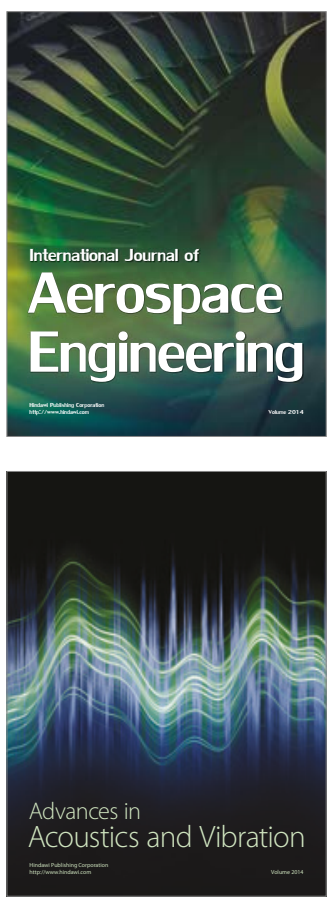

Sensor Networks 